\title{
Novel Ion Mobility Setup Combined with Collision Cell and Time-of-Flight Mass Spectrometer
}

\author{
Alexander Loboda \\ PerkinElmer SCIEX, Concord, Ontario, Canada
}

\begin{abstract}
An ion mobility cell of a novel type was coupled to an orthogonal injection time-of-flight (TOF) mass spectrometer. The mobility cell operates at low-pressure and contains a segmented RF ion guide providing an axial electric field that drives the ions towards the exit. A flow of gas is arranged inside the ion guide in such a way that the gas drag counteracts the force exerted by the axial field. Ions with different mobility coefficients can be scanned out of the ion guide by ramping the axial field strength. The ions can be analyzed intact or fragmented in a collision cell before introduction into an orthogonal TOF mass spectrometer. An ion source with matrix assisted laser desorption/ionization (MALDI) was attached to the instrument. The setup was evaluated for the analysis of peptide and protein mixture, with sequential fragmentation of multiple precursor ions from a protein digest and with mobility separation of fragment ions formed by in-source fragmentation of pure peptides. The mobility resolution for peptides was observed to be three times higher than the theoretical resolution predicted for a classical mobility setup with similar operating conditions (pressure, field strength, and length). (J Am Soc Mass Spectrom 2006, 17, 691-699) (c) 2006 American Society for Mass Spectrometry
\end{abstract}

I on mobility spectrometers [1] (IMS) are widely used in many applications as stand-alone devices. However, they become more powerful when coupled to mass spectrometers and especially time-of-flight (TOF) mass spectrometers. Recently IMS-TOF MS instruments were combined with "soft" ionization techniques such as electrospray ionization [2] (ESI) and matrix assisted laser desorption/ionization [3] (MALDI) to facilitate the analysis of biomolecules.

Various ion mobility cells can be distinctively divided into two categories: high-pressure cells and lowpressure cells. The high-pressure IMS cells operate at near atmospheric pressures of the buffer gas while the low-pressures cells operate at pressures in the region of 1 torr. High-pressure cells usually have better resolution than the low-pressure cells. This is largely because the theoretical mobility resolution depends on the voltage difference applied across the cell [4]:

$$
R_{F W H M}=\frac{1}{4 \sqrt{\ln 2}} \sqrt{\frac{q U}{k T}}
$$

Here $R_{F W H M}$ is mobility resolution at full width at half maximum (FWHM); $U$ is the voltage applied across the mobility cell; $q$ is the charge of the ion; $k$ is the Boltzmann constant; and $T$ is the temperature of the buffer gas.

Published online March 9, 2006

Address reprint requests to Dr. A. Loboda, MDS SCIEX, 71 Four Valley Drive, Concord, ON L4K 4V8, Canada. E-mail: lobodaav@sciex.com
Despite this shortcoming low-pressure mobility cells are actively being researched and developed for use in combination with mass spectrometry $[2,3,5]$. One of the reasons is that such devices are more suitable for injection of ions generated or trapped under vacuum or in low-pressure conditions.

Yet another limitation of low-pressure mobility experiments stems from the substantial diffusional spreading of the ion beam in the radial dimension, since the diffusion coefficient is inversely proportional to the pressure. Several approaches exist to overcome diffusional spreading in low-pressure mobility cells. In one approach, a radial RF field is applied to prevent radial spreading of the ions [6, 7]. Segmented quadrupole ion guides are convenient for such mobility cells. In another approach, ions are periodically refocused using electrostatic fields to keep them near the axis [8].

This report describes a novel low-pressure mobility cell with a counterflow of gas [9] that is based on a segmented RF ion guide. A flow of gas is provided in the middle portion of the ion guide to counteract the force exerted by the axial electric field. Ions become trapped at the front portion of the ion guide and can only advance to the exit when the force exerted by the electric field exceeds the drag force from the gas flow. An analogy to this setup is a wind tunnel where objects of different shapes require different pulling forces to advance in the tunnel. Ions with different mobilities can be sequentially extracted in this setup by ramping the voltage applied along the ion guide. It should be noted that gas counterflow is often used in high-pressure 


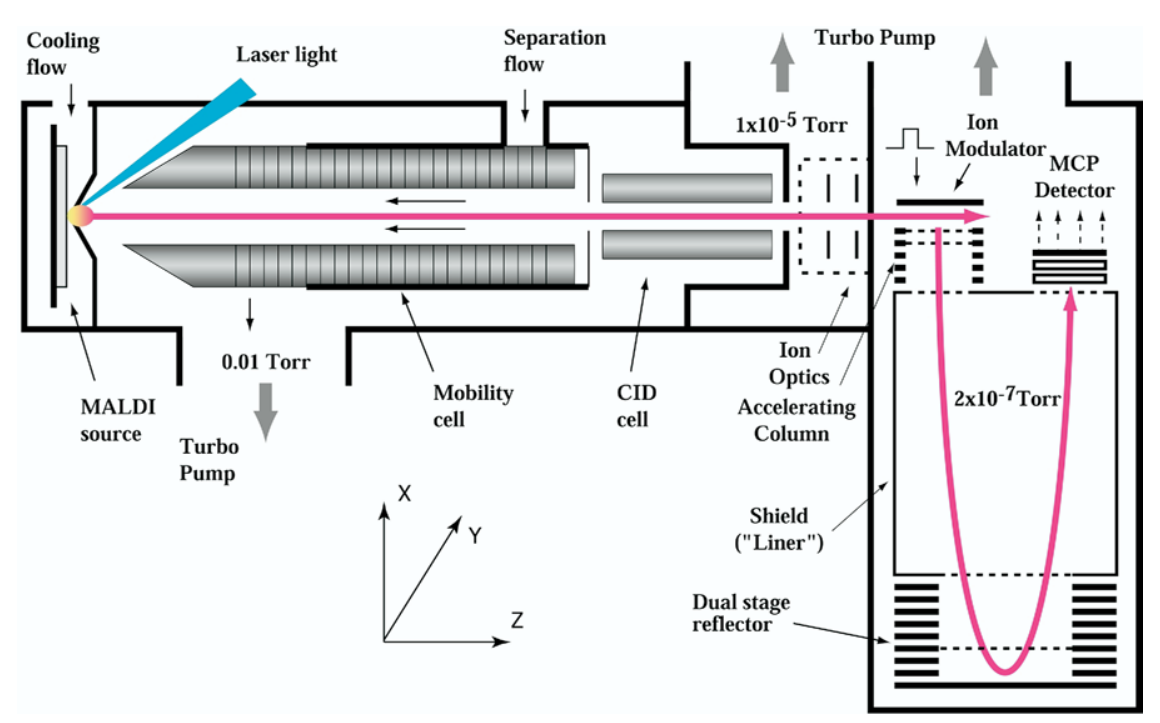

Figure 1. Diagram of mobility—time-of-flight instrument.

mobility cells to prevent contamination of the buffer gas and to reduce memory effects [10]. However, the drag force in this case is substantially smaller than the force exerted by the electrical field and therefore the ions do not become trapped but rather continue to drift at a somewhat reduced velocity.

The new ion mobility setup has several features that are advantageous over low-pressure mobility configurations. First, the RF field that provides radial confinement, combined with the electric field and gas drag that provide axial confinement, allow ions to be trapped in the ion guide for an extended period of time without losses, facilitating the efficient use of ions.

Second, the mobility peak retention times in this setup are no longer determined solely by the length of the mobility cell and the electric field applied along the axis, but to a large extent are determined by the rate at which the axial field is ramped. Therefore, the mobility spectrum is obtained over a longer period of time in comparison to a traditional mobility setup. This extension of the separation time offers a better coupling of the mobility cell to TOF instruments. Ions take about 2 ms to traverse a typical low-pressure mobility cell [11], while the typical flight time of ions in a high-performance orthogonal TOF instrument [12] is about $100 \mu \mathrm{s}$. Thus, the mobility peak will only be sampled 20 times if these two setups are combined. The problem is further amplified if a collision cell is installed between the mobility setup and the TOF analyzer. Even the fast collision induced dissociation (CID) cells equipped with auxiliary electrodes that provide axial field [13] feature transit time and ion packet broadening about a millisecond. The millisecond broadening in the CID cell does not represent a problem, however, when the mobility separation time is extended to hundreds of milliseconds or even seconds using the mobility cell described here, since the mobility peaks are correspondingly longer in time.
The third potential advantage of a mobility cell with the counterflow of gas is higher resolution. Resolution estimate based on formula (eq 1) is not applicable for this setup but it can be used as a guideline. Note that according to eq 1 , when the field strength in the mobility cell is fixed, the resolution increases as the square root of the length of the mobility cell due to the $U=E^{*} L$ relation. Since in the configuration described here, ions spend a longer time in the mobility cell, they effectively travel a longer distance, and that in turn should result in higher resolution. An estimate of resolution can be made for the case when the field is ramped relatively slowly by assuming an equilibrium distribution of ions along the axis, but even that estimate requires detailed knowledge of the drag force distribution along the axis, which was not available in this study.

An instrument incorporating the novel mobility cell had been constructed and evaluated. The instrument contains a MALDI source and mobility cell followed by collision cell and orthogonal TOF mass analyzer. The first instrument of this kind was tested at the University of Manitoba [14] by modifying an existing mobilityTOF instrument [7]. However, the gas flow in that system was not well confined, resulting in a nonuniform gas drag profile and low mobility resolution.

\section{Experimental}

\section{Ion Source and Mass Spectrometer}

The instrument employed in this study was based on the prototype of ProTOF 2000 mass spectrometer [15]. A schematic diagram of the instrument is shown in Figure 1. The collisional-focusing ion guide used in the original instrument was truncated to $200 \mathrm{~mm}$ and converted into the CID cell. Auxiliary electrodes [13] were added to the CID cell to speed up the transit of ions through 


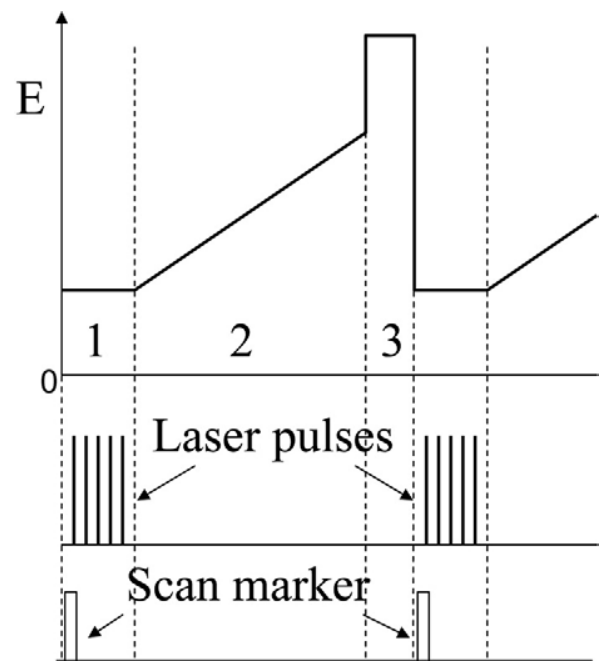

Figure 2. Mobility scan diagram. Interval 1 -laser fires at the sample, ions accumulate in the entrance part of mobility cell. Interval 2-field is ramped to effect mobility separation scan. Interval 3-axial field is set high to purge the mobility cell.

the cell. The quadrupole vacuum chamber was extended to accommodate the mobility cell.

The design of the mobility cell is based on a segmented quadrupole ion guide. The rods used in mobility cell are $346 \mathrm{~mm}$ long and $13 \mathrm{~mm}$ in diameter. The section towards the end of the mobility cell was segmented as shown in Figure 1. The segmented portion of the ion guide has 54 4-mm-long segments with a total length of $216 \mathrm{~mm}$. Ceramic inserts (140 mm long) were installed between the rods in the mobility cell to confine the flow of the separation gas. The flow of nitrogen used for mobility separation was set at $\sim 120 \mathrm{sccm}$.

The nitrogen laser in the MALDI source was replaced with a frequency tripled $\mathrm{Nd}-$ Yag solid-state laser (MALDI 200, Azura Laser, Berlin-Spandau, Germany). The laser light was delivered via $100 \mu \mathrm{m}$ fiber resulting in an elliptical spot of $100 \mu \mathrm{m} \times 115 \mu \mathrm{m}$ on the target.

The LC2Tune program for Macintosh (PerkinElmer SCIEX) was used to manipulate voltages in the quadrupole section of the instrument while time-of-flight data were collected with the TOFMA data acquisition program (TOF laboratory, University of Manitoba) using a time-to-digital converter (TDCx8, Ionwerks Houston, Texas). To synchronize laser firing, mobility scans and TOF extractions the triggering signal derived from the quadrupole control electronics was supplied to a delay generator (9650A, EG \& G, Oak Ridge, TN). One output of the delay generator was fed into channel no. 8 of the TDC and interpreted in TOFMA as a marker of the new mobility scan. The second output of the delay generator was sent to the laser. Two modes of laser operation were employed: single pulse mode when only one laser pulse was fired per mobility scan and the burst mode when laser firing was gated to allow multiple pulses per mobility scan. The TOF extraction rate was set at $9 \mathrm{kHz}$ for all experiments except the analysis of the peptide and protein mixture, where the repetition rate was set to $5 \mathrm{kHz}$.

A typical mobility scan diagram is depicted in Figure 2. During the first time interval, the laser is fired once or several times while the axial field in mobility cell is set to a low but non-zero level sufficient to trap the ions in the entrance part of the segmented section. At the beginning of this interval, a synchronization marker is sent to TDCx8. Mobility separation of ions proceeds in the second interval when the axial field is ramped up. In the last time interval (3) the axial field is set to the maximum to purge any remaining ions from the mobility cell.

\section{Sample Preparation}

Proteins and peptides with the exception of M28 peptide were purchased from $\sigma$-Aldrich. The BSA digest was obtained from Michrom Bioresources Inc. (Auburn, CA). The M28 peptide was provided by Dr. Andrej Shevchenko (Max Plank Institute, Dresden, Germany). Pre-mixed matrix solution of 4-hydroxy- $\alpha$-cyanocinnamic1 acid (4-HCCA) was purchased from Agilent Technologies (Palo Alto, CA). Stock solutions of peptides and proteins were made with acetonitrile/water 2:1 (vol/vol ratio) mixture containing $0.1 \%$ TFA. The stock solutions were mixed with matrix solution 1:3 (vol/vol) before deposition. Samples were spotted manually with deposition volume of $0.5 \mu \mathrm{l}$.

BSA samples used in the study of mobility cell and demonstration of sequential fragmentation of multiple precursor ions had $100 \mathrm{fM}$ of material deposited per spot. BSA digest peptides referred by names 927, $1439,1479,1567,1881$ have the following sequences: YLYEIAR, RHPEYAVSVLLR, LGEYGFQNALIVR, DAFLGSFLYEYSR, and RPCFSALTPDETYVPK. Pure peptides used to investigate the mobility separation of fragment ions were deposited to provide $200 \mathrm{fM}$ per spot. The M28 peptide has a sequence TTPAVLDSDGSYFLYSK. The names GluF and ACTH in the paper represent $\left[\mathrm{Glu}^{1}\right]$-fibrinopeptide $\mathrm{B}$ and adrenocorticotropic hormone fragment 18-39.

The peptide and protein mixture subjected to mobility-MS analysis had the following abbreviations (in brackets) and amounts of material per sample spot: dalargin (D), $25 \mathrm{fM}$; substance $\mathrm{P}(\mathrm{P}), 50 \mathrm{fM}$; mellitin (M), $100 \mathrm{fM}$; insulin (I), $100 \mathrm{fM}$; cytochrome $c$ (C), $400 \mathrm{fM}$; myoglobin (Myo), $200 \mathrm{fM}$.

\section{Theory}

Only a simplified theory of ion motion in the mobility section is presented here. This theory was found to be in reasonable agreement with experimental observations. Direct modeling of gas flow and ion motion in the mobility cell could be beneficial for a more detailed view and further optimization of the setup but was outside of the scope of the present work.

The simplified theory of operation of the ion mobility 
cell is based on two assumptions: constant velocity and density profiles for the buffer gas in the separation region, and a sharp decline in the gas drag near the entrance of the mobility cell. In such a configuration, when the axial field is low the ions will pile up at the entrance of the mobility cell. The axial field can then be ramped up and the ions with higher mobility will begin to overcome the drag force and move towards the exit of the mobility cell.

To perform mobility scans, electric field $E$ in the cell was ramped linearly with time $t$ according to

$$
E(t)=\frac{\Delta E}{\tau} t
$$

where $\Delta E$ describes an individual step in the ramping and $\tau$ is the time interval per individual step.

An ion will start to move towards the exit when its drift velocity induced by the electric field $v_{e l}$ matches the velocity of the buffer gas $v_{\text {gas }}$

$$
v_{e l}=v_{g a s}
$$

The drift velocity induced by the electrical field $E$ is defined by the mobility coefficient $K$ :

$$
v_{e l}=K E
$$

The threshold time interval $t_{t h}$ between the start of the ramp and the moment when the ion starts to move towards the exit can be found from eq 2 using eq 4 and taking into account condition eq 3 :

$$
t_{t h}=\frac{v_{\text {gas }} \tau}{K \cdot \Delta E}
$$

Once the ion starts to move its velocity continues to increase due to the ramping of the electric field. Therefore drift velocity $v(t)$ of the ion traveling through the separation region will be:

$$
v(t)=v_{e l}-v_{g a s}=\frac{K \cdot \Delta E}{\tau}\left(t-t_{t h}\right)
$$

Thus, the ion moves with a constant acceleration determined by the rate of ramping of the electric field. The time taken to traverse the drift region $t_{t r}$ is given by:

$$
t_{t r}=\sqrt{\frac{2 L \tau}{K \cdot \Delta E}}
$$

The total time $t_{\text {tot }}$ spent in the mobility cell can be derived from eq 5 and eq 7 as:

$$
t_{t o t}=t_{t h}+t_{t r}=\frac{v_{g a s} \tau}{K \cdot \Delta E}+\sqrt{\frac{2 L \tau}{K \cdot \Delta E}}
$$

Note that the first term in eq 8 is inversely proportional to the mobility coefficient, a behavior that is similar to the residence time in traditional mobility setups while the second term is inversely proportional to the square root of the mobility coefficient. Therefore, the relative contribution of the second term becomes smaller for slower ramping conditions. Since the relation between the total time and inverse of the mobility coefficient is nonlinear the resolution of the mobility setup

$$
R=\frac{K}{\Delta K}
$$

can no longer be calculated using

$$
R=\frac{t_{t o t}}{t_{F W H M}}
$$

where $R$ is mobility resolution (FWHM), $\Delta K$ is the FWHM spread of the measured mobility distribution for ions of a given kind, and $t_{F W H M}$ is the width (FWHM) of the mobility peak in the time domain.

However, eq 9 can be converted to:

$$
R=\frac{K}{\Delta K}=\left|\frac{K}{t_{f w h m}} \cdot \frac{d t_{\text {tot }}}{d K}\right|
$$

And, substituting eqs 5, 7, and 8 into eq 11 we obtain:

$$
R=\frac{t_{t h}+(1 / 2) \cdot t_{t r}}{t_{F W H M}}=\frac{t_{t o t}-(1 / 2) \cdot t_{t r}}{t_{F W H M}}
$$

Note that when the field ramp is slow, the contribution of $t_{t r}$ is small and the result of eq 12 approaches the result of eq 10.

Eq 8 can be used to calculate the velocity of the buffer gas $v_{\text {gas }}$ from a set of separation times measured at different ramping rates by varying time intervals $\tau$. Replacing $\tau$ with a new variable $\xi$ related to $\tau$ via

$$
\tau=\xi^{2}
$$

we obtain

$$
t_{t o t}=t_{t h}+t_{t r}=A \xi^{2}+B \xi
$$

where

$$
A=\frac{v_{g a s}}{K \cdot \Delta E}
$$

and

$$
B=\sqrt{\frac{2 L}{K \cdot \Delta E}}
$$



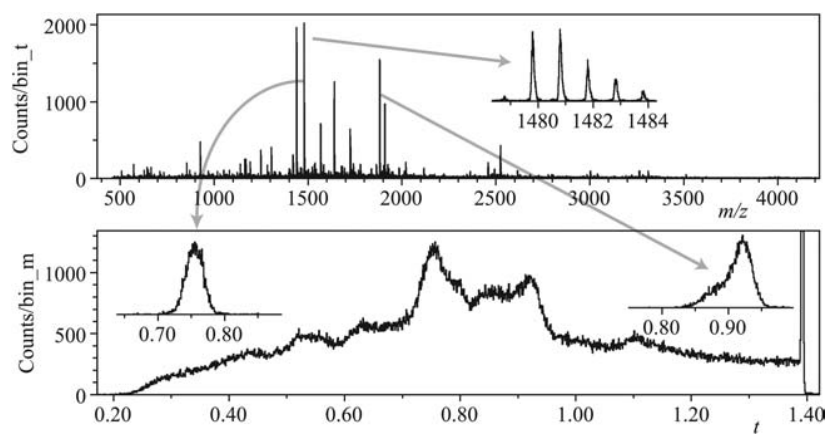

Figure 3. Mass spectrum (top) and mobility spectrum (bottom) of digested BSA sample. Inset in mass spectrum shows isotopic cluster of peptide at 1479 . Insets in mobility spectrum show extracted mobility profiles for 1479 and 1881 peaks, respectively; bin_t $=1.32 \mathrm{~ns}$; bin_m $=0.88 \mathrm{~ms}$; bin size of the inset in mass spectrum is $0.132 \mathrm{~ns}$.

tal data of total separation times acquired at different $\xi$ values. Once $A$ and $B$ are known, the velocity of the gas can be calculated from eqs 15 and 16 .

$$
v_{g a s}=2 L \frac{A}{B^{2}}
$$

\section{Results and Discussion}

\section{Study of the Mobility Cell Operation: Separation of Digest Peptides}

A BSA digest sample was used to study operation of the mobility cell with a counterflow of gas. In this study, the potential difference between the exit of mobility cell and the collision cell was set to $7 \mathrm{~V}$ to avoid fragmentation of ions exiting the mobility cell. An example of the time-of-flight and mobility spectra collected from the BSA sample is presented in Figure 3. In this case, the voltage across the segmented ion guide was ramped at a rate of $48.3 \mathrm{~V} / \mathrm{s}$. The insert in mass spectrum shows an expanded view of the isotopic cluster of peptide at 1479 Da. Note that the resolution of the TOF analyzer was not compromised by the addition of the mobility cell and remains similar to the resolution obtained with the same TOF analyzer before modification [15]. Moreover the same ion counting TOF detection system was still capable of recording the spectra without peak saturation. To reduce the probability of peak saturation, only one laser pulse was fired per extraction in this experiment.

Insets in the mobility scan spectrum show extracted ion intensity distributions versus mobility scan time for peptides at 1479 and $1881 \mathrm{Da}$, respectively. Since the mobility voltage ramp starts at a non-zero value, the mobility residence time has to be corrected by adding an offset time to match $t_{\text {tot }}$ definition eq 8 taking into account eq 2. In the example of Figure 3, the offset time was $0.420 \mathrm{~s}$. Other factors that contribute to the mobility peak time distribution are related to the travel times through the CID cell and through the TOF mass analyzer. Both of these contributions are on the millisecond or sub-millisecond scale and were assumed to be negligible in this study.

The mobility peak corresponding to the ions of the peptide at $1881 \mathrm{Da}$ shows significant tailing. Similar tailing is commonly observed in traditional mobility measurements indicating presence of isomers with different shapes [16]. The problem with such tailing can be reduced when the mobility cell is heated to anneal the ions [17]. However, heating was not employed in the current study.

A series of mobility-MS spectra were collected for BSA digest sample at various scan rates to compare the results with the predictions of the simplified theory. The series was collected with step size of $\Delta E=0.659$ $\mathrm{V} / \mathrm{m}$ and for a set of time intervals $\tau=0.24,0.6,1.2,3$, and 9 ms. Mobility separation times and peak widths were measured for three peptides 927, 1479, and 1567 that exhibited the least amount of tailing. The mobility separation times as functions of $\xi=\tau^{1 / 2}$ are plotted in Figure 4 . The experimental data were fitted with the second-order polynomial function according to eq 14, allowing calculation of $\mathrm{A}$ and $\mathrm{B}$, and therefore the gas velocity from eq 17 . The gas velocity calculated for the three ions gave somewhat different values. To overcome the problem the $A$ coefficients were slightly adjusted to values $(388.00 ; 377.11 ; 299.35)$ leading to the corresponding change in $B$ coefficients, so that the gas velocities calculated using eq 17 would match each other for all three ions. The velocity of the buffer gas calculated in this way is $v_{\text {gas }}=145 \mathrm{~m} / \mathrm{s}$. That relatively high value, approximately one-third of the speed of sound, appears to be reasonable taking into account the gas flow rate, expected pressure in the mobility cell, and its dimensions.

The pressure of the buffer gas in the mobility cell can be also estimated from the velocity of the buffer gas, the

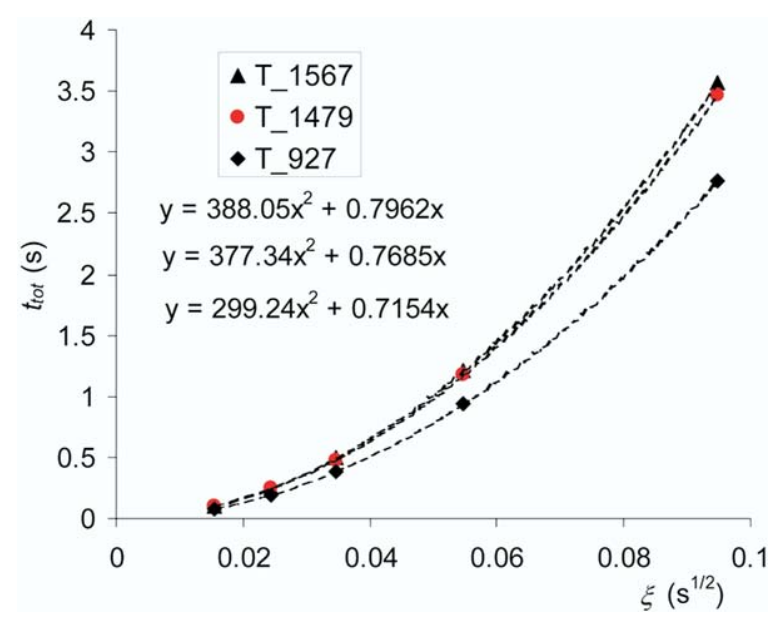

Figure 4. Mobility separation times as functions of $\xi$ for three peptides, 1567, 1479, and 927. Dashed lines show best-fit secondorder polynomial functions for each peptide. Equations in the graph describe the best-fit functions. 
Table 1. Resolution in mobility cell as a function of $\tau$ for three peptides $-927,1479$, and 1567

\begin{tabular}{rccccc}
\hline Peak & $0.24 \mathrm{~ms}$ & $0.6 \mathrm{~ms}$ & $1.2 \mathrm{~ms}$ & $3 \mathrm{~ms}$ & $9 \mathrm{~ms}$ \\
\hline \hline 927 & 22 & 27 & 28 & 34 & 40 \\
1479 & 22 & 29 & 29 & 33 & 39 \\
1567 & 25 & 29 & 31 & 34 & 38 \\
\hline
\end{tabular}

field strength at the threshold time $t_{t h}$, and a typical reduced mobility coefficient $K$ for peptides of interest by rearranging the following formula:

$$
v_{\text {gas }}=K E=K_{r} \frac{P_{a t m}}{P} \frac{\Delta E}{\tau} t_{t h}
$$

where $P$ is the pressure in the mobility cell and $P_{a t m}=$ 760 torr is atmospheric pressure. This leads to a pressure estimate in collision cell of $\sim 50$ mTorr. The pressure inside the mobility cell was not measured directly. The pressure outside the mobility cell was measured to be 10 mTorr as indicated on Figure 1.

Mobility resolutions calculated according to eq 12 for scans with different $\tau$ values are summarized in Table 1 . As expected, the resolution goes up when the rate of ramping goes down due to an increase in the effective distance the ions travel while moving through the buffer gas. However, the resolution increase was not as high as the square root of the length predicted by eq 1 , taking into account $U=E^{*} L$ for traditional mobility cells.

It is interesting to compare the resolution of this new mobility technique with the theoretical resolution of a traditional mobility cell of the same length, assuming the field strength is equal to the field strength in the new cell at the threshold time $t_{t h}$. Eq 1 with $U$ defined from experimental parameters for the peak of peptide at $1567 \mathrm{Da}$

$$
U=L \frac{\Delta E}{\tau} t_{t h}
$$

gives a resolution of $\cong 11$. The new mobility technique produces a resolution of $\sim 39$ for the same length, pressure, and separation field, providing a significant improvement in performance.

Just as in traditional mobility cells, the resolution of this mobility cell with counterflow of gas can be further improved by increasing its operating pressure. Segmented quadrupole ion guides have been demonstrated to operate at pressures exceeding 1 torr [18]. Thus, with an appropriate pumping arrangement, the operating pressure of the mobility cell with the counterflow of gas could be brought up from the 50 mTorr used in this study to about 1 torr. That, in turn, should result in a 4-fold gain in resolution assuming it follows the pressure dependence known in traditional mobility cells. Such resolution of $\sim 150$ (FWHM), if achieved, will be on par with the resolution observed in high-performance, high-pressure mobility cells, but with an additional benefit of operating the cell at low-pressure, which allows more efficient coupling with the mass spectrometer.

\section{Sequential Fragmentation of Multiple Precursor Ions}

The ions exiting the mobility cell can be fragmented in the CID cell by maintaining sufficient potential difference between the exit of the mobility cell and the entrance of the CID cell. In this mode of operation, multiple precursor ions can be fragmented and recorded sequentially. This is strikingly different from a typical MS/MS operation in a tandem mass spectrometer such as QqTOF [12]. In the QqTOF, only one precursor ion can be selected for fragmentation while the rest of the ions are rejected in the Q1 mass filter. Therefore, the efficiency of QqTOF in MS/MS mode is inversely proportional to the number of precursor ions of interest present in the sample. Such a situation is quite common, for example, in the case of protein digest samples that contain many peptides where more than one of them may require an in-depth analysis via CID.

The BSA digest sample was used to study sequential fragmentation of multiple precursor ions in the IM-CIDTOF mode of operation. Mobility scan conditions were $\Delta E=0.659 \mathrm{~V} / \mathrm{m}$ and $\tau=1.2 \mathrm{~ms}$. A burst of six laser pulses for each mobility scan was employed in this experiment. The use of the burst mode to collect fragment ion spectra did not lead to peak saturation in TOF

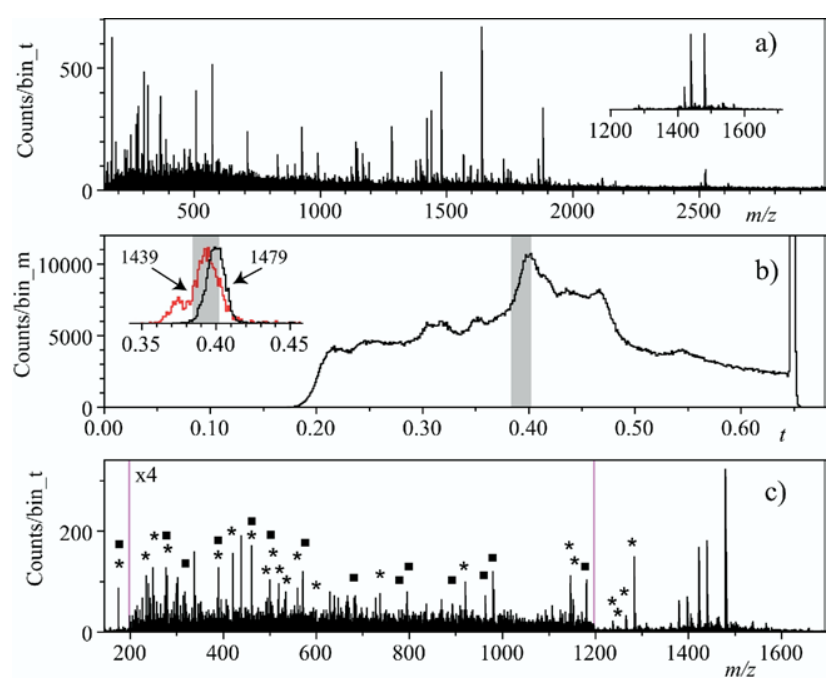

Figure 5. Mobility and mass spectra produced in "sequential fragmentation of multiple precursor ions" experiment. (a) Combined mass spectrum; (b) combined mobility spectrum; (c) mass spectrum extracted from the mobility region depicted on (b) by gray rectangle. Inset in (a) shows section of mass spectrum extracted from a mobility region depicted by gray rectangle but in the experiment when the CID energy was set to $7 \mathrm{~V}$. Inset in (b) shows extracted mobility profiles for peaks of 1439 and 1479 peptides; bin_t $=0.264$ ns; bin_m $=1.1 \mathrm{~ms}$. 
since the intensity of each precursor ion is redistributed across a wide range of fragment ions. During each mobility scan, the CID energy was ramped linearly with time to follow the increase in the mass of precursor ions. The CID energy value at the beginning of the ramp and the rate of its ramping were manually adjusted to effect the desired degree of fragmentation. Optimal results were obtained when CID energy was ramped from 10 to $230 \mathrm{~V}$. The data were collected for $100 \mathrm{~s}$ with total of about 150 mobility scans and 900 laser shots fired at the sample.

The results of this experiment are shown in Figure 5. Figure 5a shows a combined mass spectrum that contains peaks of all precursor and fragment ions. Figure $5 \mathrm{~b}$ shows the behavior of the ion current as a function of mobility scan time. The inset of Figure $5 b$ shows ion current behavior for precursor ions of peptides at 1439 (left) and 1479. The intensities of the peaks were normalized to the maximum. Grey rectangles in Figure $5 \mathrm{~b}$ indicate the area on the mobility scale from which the TOF spectrum shown in Figure $5 c$ was extracted. The inset in Figure 5a illustrates the quality of precursor ion selection in the mobility cell. The mass spectrum in the inset was extracted over the same area on the mobility scale from the data acquired when the CID energy was set to $7 \mathrm{~V}$. Two major precursor peaks at 1439 and 1479 and a few minor components are present, and were fragmented simultaneously, resulting in the spectrum shown in Figure 5c. Fragment peaks from extracted spectrum were used to search NCBI database with the MS-Tag search engine [19]. The search parameters were the following: mass accuracy of precursor ion, $20 \mathrm{ppm}$; mass accuracy of fragment ions, $40 \mathrm{ppm}$; instrument type, "MALDI-TOF". In the first search, the mass of the precursor ion was taken from the measurement of the mass of 1439 peak and in the second search precursor ion mass was taken from the 1479 peak. In both searches, peptides were unambiguously identified. Fragment ion peaks assigned by MS-Tag as $a, b, y$, and internal fragment ions are indicated in Figure $5 c$ as stars for 1439 precursor ion and solid squares for 1479 precursor ion. Standard deviation of mass error for all fragment ions is $13 \mathrm{ppm}$.

\section{Mobility Separation of $a, b$, and y Fragment Ions from Pure Peptides}

Mobility separation of peptide fragment ions was performed to test the hypothesis that there is a significant difference in mobility between fragments of different types such as $b$ and $y$ ions. Such differences could be useful when de novo sequencing of a peptide from its fragments is performed. Three peptides, M28, GluF, and $\mathrm{ACTH}$, were used in this experiment. Peptides were fragmented in the ion source before the mobility separation region. CID fragmentation was effected by providing a high potential difference between the cone
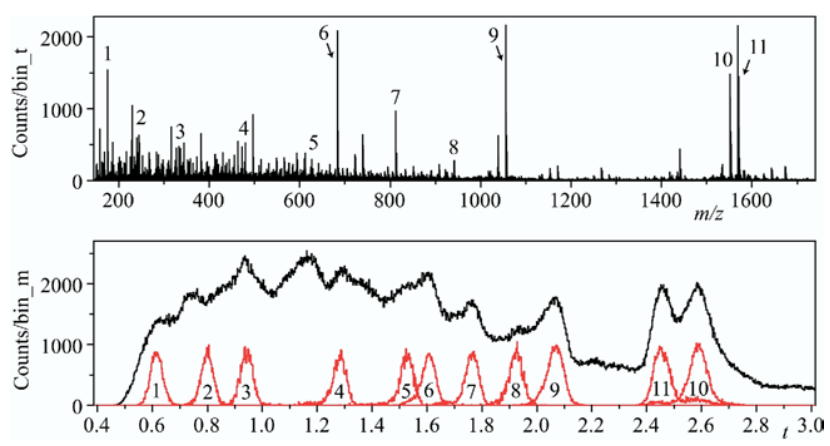

Figure 6. Mass spectrum (top) and mobility spectrum (bottom) of GluF peptide fragmented before mobility separation. For explanation of peak labeling see paper. Labeled peaks in mobility spectrum show mobility profiles extracted for each of the labeled mass spectral peaks. Extracted mobility peaks are normalized for convenience; bin_t $=0.264 \mathrm{~ns}$; bin_m $=2.2 \mathrm{~ms}$.

and the segmented ion guide, similar to the method described in [20].

The mass and mobility spectra of GluF fragments are shown in Figure 6. Labels 1 to 9 are assigned to $y$ series ions from $y_{1}$ to $y_{9}$. The protonated ion of intact GluF is designated as 11 while the fragment ion that represents the protonated GluF ion with the loss of $\mathrm{H}_{2} \mathrm{O}$ is labeled as 10. Grey peaks shown underneath the mobility spectrum correspond to extracted mobility traces for the labeled ions. For convenience, these traces were normalized in intensity. Note that the order of the $y$ series ions in the mobility separation panel coincides with the order with which these ions appear in the mass spectrum, while the order of appearance of peaks 10 and 11 is reversed. The reversal of the order for peaks 10 and 11 is noteworthy, considering the small difference in mass and composition between the two ions. It can be speculated that the difference in mobility stems from a significant change in the shape of the ion triggered by the loss of a water molecule.

Figure 7 shows mobility separation time converted into $t_{t h}$ versus mass to charge ratio for fragment ions of M28, GluF, and ACTH. Circles and squares correspond to $b$ and $y$ ions, respectively. No significant correlation between the type of the ion and its mobility was observed for these fragments. Fragment ions of other types such as $a, b-17 \mathrm{Da}$, and $y-17 \mathrm{Da}$ were also present. All identified fragment ions are depicted in Figure 7 marked with " $x$ ", and their positions correlate quite well with the function $t_{t h}=0.0668(\mathrm{~m} / \mathrm{z})^{0.518}$.

Fragment ions separated by the mobility cell can be further fragmented in the CID cell using the method described in the previous section. In the situation when only one component is present in the sample, this will generate pseudo-MS ${ }^{3}$ spectra but with higher efficiency than traditional tandem mass spectrometers. Indeed, even if only one component of interest is present in the sample, it results in multiple components when fragmented, and selection of one of these fragments leads to the loss of other fragments during $\mathrm{MS}^{3}$ cycle of traditional tandem mass spectrometers. 


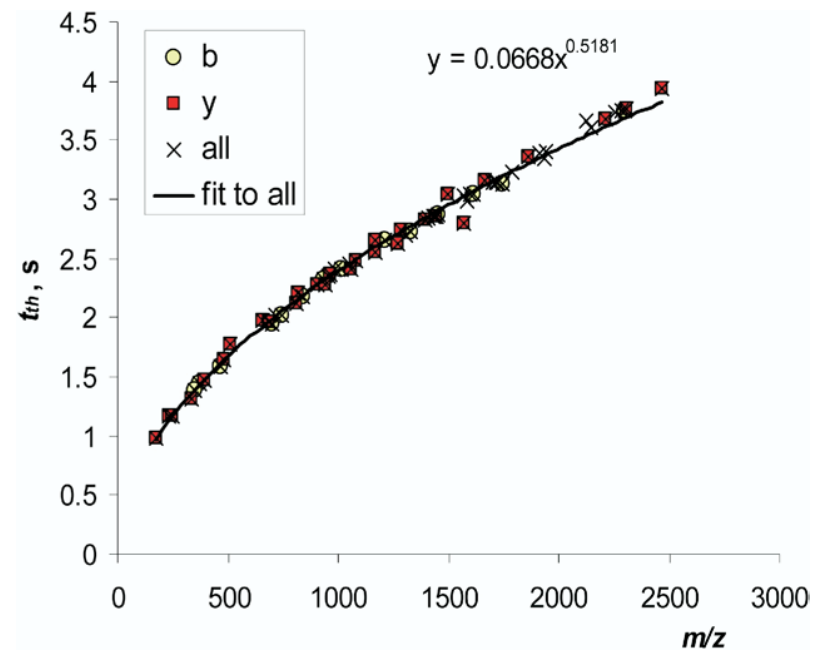

Figure 7. Threshold times derived from experimental data for fragment ions as a function of their mass to charge ratio. Square represents $b$-ions, circle represents $y$-ions, and " $x$ " represents all identified fragment ions. Solid line shows best-fit power function. Equation in the graph describes the best-fit function.

\section{Mobility MS Analysis of Protein and Peptide Mixture}

Figure 8 shows mass and mobility spectra of the peptide and protein mixture recorded to test the mass range capabilities of the setup. Only one setting of the RF voltages and one axial field ramping profile in the mobility cell were used in the experiment. The mass range covered by this experiment is about a factor of 20 . If an extension of the mass range is required, another setting can be interleaved to facilitate transmission of higher or lower mass ions. For example, lower RF voltages and axial field were used in the experiments with mobility separation of lighter fragment ions of peptides.

Multiply charged ions that are commonly seen in MALDI of proteins were also observed in this experiment for cytochrome $c$ and myoglobin. The difference in mobility of multiply charged ions was more than sufficient for complete separation of doubly charged ions from singly charged ions with the same mass to charge ratio. Figure $8 \mathrm{c}$ shows the extracted mass spectrum from the region in the mobility spectrum designated by the gray rectangle in Figure $8 \mathrm{~b}$. The doubly charged ions of cytochrome $c$ appear around $m / z 6000$, while the singly charged ions representing other components in the mixture appear at $\mathrm{m} / \mathrm{z}$ around 4000 .

\section{Conclusions and Future Outlook}

A tandem instrument incorporating a novel mobility cell and time-of-flight mass analyzer was built and evaluated. Operation of the mobility cell was characterized experimentally and with the help of simplified theory. Good agreement between the theory and experiment was observed. The new mobility cell offers a significant improvement in resolution in comparison to traditional mobility cells of similar dimensions and operating pressure. Longer separation times in the new mobility cell facilitate better coupling to CID cell and a TOF mass analyzer. Resolution or transmission efficiency of the TOF mass analyzer was not compromised by the addition of the mobility cell.

The resolution of the mobility cell can be further improved by redesigning it to operate at higher pressure. Gas dynamic modeling will likely be required. It appears feasible to obtain resolution of 150 (FWHM) for the low-pressure mobility cell of manageable dimensions operating at a pressure of about 1 torr.

Instrument operation with sequential fragmentation of multiple precursor ions was demonstrated. This mode facilitates efficient use of ions when analyzing samples containing more than one component of interest. The two-dimensional dataset collected in this experiment can be processed off-line to provide precursor ion scans, fragment ion scans, neutral loss scans, and other types of one dimensional scans commonly used in tandem mass spectrometers. The low-resolution of precursor ion selection in mobility cell can be somewhat offset by the high-resolution and mass accuracy provided by TOF mass analyzer.

The mobility separation of peptide fragment ions did not reveal significant difference between $\mathrm{C}$ and $\mathrm{N}$ terminus ions ( $b$ and $y$ fragments), and did not point to additional sequence specific information unavailable from mass spectra. However, mobility separation with higher resolution might still be able to identify differences. When only one component is present in the sample, sequential fragmentation of multiple precursor ions can be extended to provide pseudo-MS ${ }^{3}$ informa-
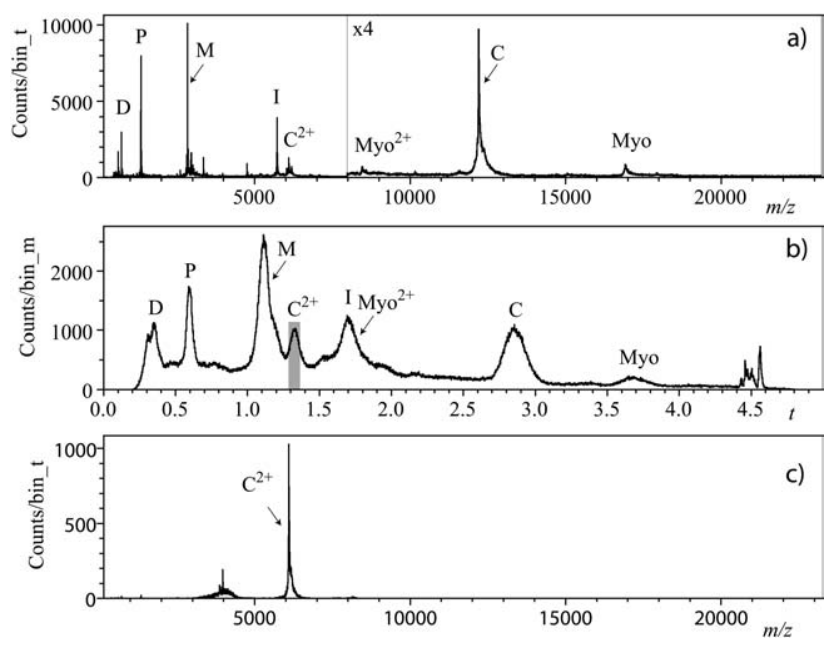

Figure 8. Mobility and mass spectra of protein and peptide mixture. (a) Shows combined mass spectrum; (b) shows combined mobility spectrum; (c) shows mass spectrum extracted from the region depicted by gray rectangle in the mobility spectrum. Peaks are labeled according to the following: $\mathrm{D}$, dalargin; $\mathrm{P}$, substance $\mathrm{P}$; $\mathrm{M}$, mellitin; I, insulin; C, cytochrome $c$; Myo, myoglobin; $\mathrm{C}^{2+}$, doubly charged ion of cytochrome $c$; $\mathrm{Myo}^{2+}$, doubly charged ion of myoglobin; bin_t $=26.4 \mathrm{~ns}$; bin_m $=2.0 \mathrm{~ms}$. 
tion with highly efficient use of ions. The method could be further enhanced if a quadrupole mass filter is added between the ion source and the mobility cell to select precursor ions.

An interesting extension of the separation capabilities of the mobility cell with counterflow of gas could be provided by the use of an axial field with superimposed time-dependent asymmetric waveform. Currently, high-field asymmetric waveform ion mobility spectrometry (FAIMS) [21, 22] is only available in highpressure instruments. In a simplified view, this method separates ions on the basis of nonlinear behavior of their mobility at high fields, providing information distinctively different from the mobility coefficient. Much lower field is required to achieve the nonlinear behavior of ion mobility at lower pressure. Therefore, nonlinear effects in the low-pressure cell can be intensified with respect to high-pressure cells. The separation in the mobility cell with counterflow of gas would then be governed by three parameters: gas flow rate, electric filed strength, and the amplitude of the asymmetric waveform, enabling control of the degree of FAIMS and mobility separation and providing a combination of both techniques in one device. An additional advantage of the proposed setup over FAIMS instruments in practice today is potentially higher efficiency. Modern FAIMS setups transmit only one group of ions at a time, simultaneously rejecting the rest of the ions while the proposed setup will store all the ions and eject them sequentially, thereby reducing ion losses.

\section{Acknowledgments}

This research work was supported by Genome Canada through the Genome Prairie's Enabling Technologies Project. The author thanks his colleagues at SCIEX-Bruce Thomson, Igor Chernushevich, and Suzanne Ackloo for help and useful discussions. He thanks Milorad Bosnjakovic and Tiberiu Gera for the help with mechanical and electrical design of mobility cell. A special credit goes to Werner Ens and Vic Spicer from the University of Manitoba who generously provided a modified version of their TOFMA program used in data acquisition and processing.

\section{References}

1. Hill, H. H.; Jr.; Siems, W. F.; St. Louis, R. H.; McMinn, D. G. Ion Mobility Spectrometry. Anal. Chem. 1990, 62(23), 1201A-1209A.

2. Hoaglund-Hyzer, C. S.; Lee, Y. J.; Counterman, A. E.; Clemmer, D. E. Coupling Ion Mobility Separations, Collisional Activation Techniques, and Multiple Stages of MS for Analysis of Complex Peptide Mixtures. Anal. Chem. 2002, 74(5), 992-1006.

3. Gillig, K. J.; Ruotolo, B.; Stone, E. G.; Russell, D. H.; Fuhrer, K.; Gonin, M.; Schultz, A. J. Coupling High-Pressure MALDI with Ion Mobility/ Orthogonal Time-of-Flight Mass Spectrometry. Anal. Chem. 2002, 72(17), 3965-3971.

4. Revercomb, H. E.; Mason, E. A. Theory of Plasma Chromatography/ Gaseous Electrophoresis. Anal. Chem. Rev.]?1975, 47/sb:volume-nr7), 970-983.

5. Wyttenbach, T.; Kemper, P. R.; Bowers, M. T. Design of a New Electrospray Ion Mobility Mass Spectrometer. Int. J. Mass Spectrom. 2001, 212, 13-23.

6. Javahery, G.; Thomson, B. A. A Segmented Radiofrequency-Only Quadrupole Collision Cell for Measurements of Collision Cross Section on a Triple Quadrupole Mass Spectrometer. J. Am. Soc. Mass Spectrom. 1997, 8(7), 697-702.

7. Kozlovski, V. I.; Loboda, A. V.; Spicer, V.; McNabb, J.; Ens, W.; Standing, K. G. Mobility Separation of MALDI and ESI Ions in a Segmented Quadrupole Ion Guide Coupled to an Orthogonal TOF MS. Proceedings of the 50th ASMS Conference on Mass Spectrometry and Allied Topics; June 2002; Extended Abstracts.

8. Gillig, K. J., Russell,, D. H. Periodic Field Focusing Ion Mobility Spectrometer. U.S. patent, 2001, 6639213.

9. Loboda,, A. V. Setup for Mobility Separation of Ions Implementing an Ion Guide with an Axial Field and Counterflow of Gas. U.S. patent 2002, 6630662.

10. Baim, A. M.; Hill, H. H., Jr. Tunable Selective Detection for Capillary Gas Chromatography by Ion Mobility Monitoring. Anal. Chem. 1982, 54, $38-43$.

11. Ruotolo, B. T.; McLean, J. A., Gillig, K. J.; Russell, D. H. The Influence and Utility of Varying Field Strength for the Separation of Tryptic Peptides by Ion Mobility-Mass Spectrometry. J. Am. Soc. Mass Spectrom. 2005, 16(2), 158-165.

12. Chernushevich, I. V.; Loboda, A. V.; Thomson, B. A. An Introduction to Quadrupole Time-of-Flight Mass Spectrometry. J. Mass Spectrom. 2001, 36, 849-865.

13. Loboda, A.; Krutchinsky, A.; Loboda, O.; McNabb, J.; Spicer, V.; Ens, W.; Standing, K. G. Novel LINAC II Electrode Geometry for Creating an Axial Field in a Multipole Ion Guide. Eur. J. Mass Spectrom. 2000, 6(6), 531-536.

14. Serov, A.; Kozlovski, V. I.; Loboda, A. V.; Spicer, V.; Ens, W.; Standing, K. G., unpublished.

15. Loboda, A. V.; Ackloo, S.; Chernushevich, I. V. A High-Performance Matrix-Assisted Laser Desorption/Ionization Orthogonal Time-ofFlight Mass Spectrometer with Collisional Cooling. Rapid Commun. Mass Spectrom. 2003, 17, 2508-2516.

16. Dugourd, P.; Hudgins, R. R.; Clemmer, D. E.; Jarrold, M. F. HighResolution Ion Mobility Measurements. Rev. Sci. Instrum. 1997, 68(2), 1122-1129.

17. Clemmer, D. E.; Jarrold, M. F. Ion Mobility Measurements and Their Applications to Clusters and Biomolecules. J. Mass Spectrom. 1997, 32, 577-592.

18. Dodonov, A.; Kozlovski, V.; Loboda, A.; Raznikov, V.; Sulimenkov, I.; Tolmachev, A.; Kraft, A.; Wollnik, H. A New Technique for Decomposition of Selected Ions in Molecule Ion Reactor Coupled with OrthoTime-of-Flight Mass Spectrometry. Rapid Commun. Mass Spectrom. 1997, 11, 1649-1656.

19. http://prospector.ucsf.edu

20. Ackloo, S.; Loboda, A. V. Applications of a Matrix-Assisted Laser Desorption/Ionization Orthogonal Time-of-Flight Mass Spectrometer. 1. Metastable Decay and Collision-Induced Dissociation for Sequencing Peptides. Rapid Commun. Mass Spectrom. 2005, 19, 1-8.

21. Buryakov, I. A.; Krylov, E. V.; Nazarov, E. G.; Rasulev, U. Kh. A New Method of Separation of Multi-Atomic Ions by Mobility at Atmospheric Pressure Using a High-Frequency Amplitude-Asymmetric Strong Electric Field. Int. J. Mass Spectrom. Ion Processes. 1993, 128, 143-148.

22. Purves, R. W.; Guevremont, R.; Day, S.; Pipich, C. W.; Matyjaszczyk, M. S. Mass spectrometric characterization of a high-field asymmetric waveform ion mobility spectrometer. Rev. Sci. Instrum. 1998, 69(12), 4094-4105. 